\title{
THE MODEL COMPANION OF THE THEORY OF COMMUTATIVE RINGS WITHOUT NILPOTENT ELEMENTS
}

\author{
L. LIPSHITZ AND D. SARACINO
}

Abstract. We show that the theory of commutative rings without nilpotent elements has a model companion. The model companion is decidable and is the model completion of the theory of commutative regular rings.

Recall that a theory $K$ is model-complete if for any model $M$ of $K$, $K \cup D(M)$ is complete, where $D(M)$ denotes the diagram of $M$. A natural generalization of this notion is that of a model completion. We say that $K^{\prime}$ is a model completion of $K$ if $K^{\prime}$ extends $K$ and, for any model $M$ of $K, K^{\prime} \cup D(M)$ is consistent and complete (see [5]). For example the theory of algebraically closed fields is a model completion of the theory of fields and the theory of real closed fields is a model completion of the theory of ordered fields.

A further generalization is the idea of a model companion. We say that $K$ and $K^{\prime}$ are mutually model consistent if every model of $K$ can be embedded in a model of $K^{\prime}$ and vice versa. $K^{\prime}$ is a model companion of $K$ if $K$ and $K^{\prime}$ are mutually model consistent and $K^{\prime}$ is model-complete.

Model completions and model companions (when they exist) are unique. For this and other elementary properties see [5] and [6].

In everything that follows we shall use the word ring to mean ring with identity. We call a ring $R$ regular (in the sense of von Neumann) if for any $x \in R$ there exists $y \in R$ such that $x y x=x$. (A good general reference for the algebra relevant to this paper is [3].) Notice that in any commutative ring the set of idempotents forms a Boolean algebra under the operations $e \cup f=e+f-e f$, $e \cap f=e f$. Hence when we say that $e$ is a subidempotent of $f$ we mean that $e f=e$ (i.e. $e \cap f=e$ ). $e$ is a minimal idempotent if $e f=f$ implies that $f$ is either $e$ or 0 . We shall say that a quantifierfree formula $\psi\left(a_{1}, \cdots, a_{n}\right)$ holds on an idempotent $e$ of a ring $R$ if the formula obtained from $\psi$ by multiplying every term in $\psi$ by $e$ holds in $R$.

Received by the editors February 25, 1972 and, in revised form, July 28, 1972.

AMS (MOS) subject classifications (1970). Primary 02H05, 02H99; Secondary 02G05, 02G20.

Key words and phrases. Model companion, model completion, amalgamation property, rings without nilpotent elements, decidable theory of rings.

${ }^{1}$ Supported by a National Science Foundation Graduate Fellowship. 
Using nilpotent elements G. Cherlin has recently shown that the theory of commutative rings has no model companion. We shall show that the existence of nilpotents is the only obstacle, i.e. that the theory of commutative rings without nilpotent elements has a model companion. We are able to write down a set of axioms for this model companion; we also show that it is the model completion of the theory of commutative regular rings. It has been brought to our attention that this answers a question posed in [7].

Definition. Let $K$ denote the theory of commutative rings without nilpotent elements. Let $K^{\prime}$ have the following axioms:

(i) the axioms of $K$,

(ii) the axiom of regularity, i.e. $\forall x \exists y(x y x=x)$,

(iii) a statement that there are no minimal idempotents,

(iv) a set of statements saying that every monic polynomial has a root.

THEOREM 1. $K^{\prime}$ is the model companion of $K$.

Proof. First we establish the mutual model consistency of $K$ and $K^{\prime}$. Since $K^{\prime}$ extends $K$ it suffices to show that any model of $K$ can be embedded in a model of $K^{\prime}$. Let $R$ be a model of $K$. Since $R$ has no nilpotents we know that the intersection of all prime ideals $P$ of $R$ is trivial (see [3]). Hence we have an embedding $R \rightarrow \prod_{P} R / P \rightarrow \prod_{P} F_{P}$, where $P$ varies over all prime ideals of $R$ and $F_{P}$ is the quotient field of $R / P$. Thus we clearly have an embedding $R \rightarrow \prod_{i \in I} F_{i}$ where each $F_{i}$ is an algebraically closed field, for some index set $I$. For each $i \in I$ let $X_{i}$ be a copy of the Cantor space, and let $C_{i}$ be the set of locally constant functions from $X_{i}$ to $F_{i}$. (We say $f: X \rightarrow F$ is locally constant if for each $x \in X$ there is an open set in $X$ which contains $x$ and on which $f$ is constant.) Let $M=\prod_{i \in I} C_{i}$. There is a natural embedding $F_{i} \rightarrow C_{i}$ and hence an embedding of $R$ into $M$. It is not difficult to check that each $C_{i}$ is a model of $K^{\prime}$ and that consequently $M$ is a model of $K^{\prime}$.

Next we must show that $K^{\prime}$ is model-complete. To do this we shall use Robinson's test (see [5]). Let $A \subset B$ be two models of $K^{\prime}$. Consider a primitive formula

$$
\varphi\left(a_{1}, \cdots, a_{n}\right)=\exists x_{1} \cdots \exists x_{k}\left(\bigwedge_{j=1}^{r} \varphi_{j} \wedge \bigwedge_{m=1}^{s} \psi_{m}\right),
$$

where the $a_{i}$ are in $A$, each $\varphi_{j}$ is an equality and each $\psi_{m}$ is an inequality. Assume $B \vDash \varphi$; we must show $A \vDash \varphi$. We will do this in two steps, the first using axioms (ii) and (iv), the second axioms (ii) and (iii).

As above we can embed $B$ in the product $\prod B / P$, where $P$ varies over $\operatorname{Spec}(B)$, the set of prime ideals of $B$. Notice that since $\bigcap_{P}(A \cap P)=\{0\}$ we can embed $A$ into $\prod A /(A \cap P)$, and in fact we have a commutative 
diagram

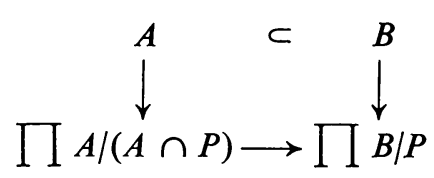

Now $\prod B / P \vDash \varphi$. But each $A /(A \cap P)$ and $B / P$ is a field because every prime ideal in a commutative regular ring is maximal [3], and is in fact algebraically closed by axiom (iv). Therefore by the model-completeness of the theory of algebraically closed fields [5], $\prod A /(A \cap P) \vDash \varphi$. For if $b_{1}, \cdots$, $b_{k}$ in $\prod B / P$ satisfy $\bigwedge_{j=1}^{r} \varphi_{j} \wedge \bigwedge_{m=1}^{s} \psi_{m}$, we can, by this model-completeness, let $a_{1}, \cdots, a_{n}$ in $\prod A /(A \cap P)$ be such that, modulo any $P$ in $\operatorname{Spec}(B)$, $a_{1}, \cdots, a_{k}$ satisfy the same $\varphi_{j}$ 's and $\psi_{m}$ 's as do $b_{1}, \cdots, b_{k}$; then clearly

$$
\prod A /(A \cap P) \vDash\left(\bigwedge_{j=1}^{r} \varphi_{j} \wedge \bigwedge_{m=1}^{s} \psi_{m}\right)\left(a_{1}, \cdots, a_{k}\right) .
$$

This completes the first part of the proof; axiom (iv) has done its work, and we will not use it again.

The second part of the proof consists of showing that $\prod A /(A \cap P) \vDash \varphi$ implies $A \vDash \varphi$. Before going on with this, we state the following

Lemma. Consider $A$ as a subring of $\prod A /(A \cap P)$, as above. For any open sentence $\chi\left(a_{1}, \cdots, a_{n}\right)$ defined in $A$ there exists an idempotent in $A$ on which $\chi$ holds identically and on whose complement $\neg \chi$ holds identically. (Any idempotent e in $\prod A /(A \cap P)$ has either a zero or a one corresponding to each factor in the product. When we say $\chi$ holds identically on $e$, we mean that $\chi$ holds at each factor where e takes the value 1.)

Proof. Since the idempotents in $A$ form a Boolean algebra, it suffices to prove the result for atomic statements.

Consider $p\left(a_{1}, \cdots, a_{n}\right)=q\left(a_{1}, \cdots, a_{n}\right)$, where $p$ and $q$ are polynomials. Since $A$ is regular there is an $x$ in $A$ such that $(p-q) x(p-q)=p-q$. Let $f=x(p-q)$. Then $f f=x(p-q) x(p-q)=x(p-q)=f$. Let $e=1-f$. Then $e e=e$. Now $(p-q) e=(p-q)(1-f)=(p-q)-(p-q)=0$. Therefore $p=q$ holds identically on $e$. On $f=1-e, p\left(a_{1}, \cdots, a_{n}\right) \neq q\left(a_{1}, \cdots, a_{n}\right)$ holds identically, since $(p-q) x f=f f=f$.

Now suppose $\prod A /(A \cap P) \vDash \varphi$; to show $A$ k $\varphi$ we claim first that it suffices to consider the case where $\varphi$ contains only one negation, i.e. $s=1$. For if $\theta_{m}=\bigwedge_{j=1}^{r} \varphi_{j} \wedge \psi_{m}$, then for any $m, 1 \leqq m \leqq s, \prod A /(A \cap P) \vDash$ $\exists x_{1} \cdots \exists x_{k} \theta_{m}$, whence by the assumption of the claim $A \vDash \exists x_{1} \cdots \exists x_{k} \theta_{m}$. Let $c_{m, 1}, \cdots, c_{m, k}$ satisfy $\theta_{m}$ in $A$. Then, by the Lemma, $\theta_{m}\left(c_{m, 1}, \cdots, c_{m, k}\right)$ holds identically on some idempotent $e_{m}$ in $A$, and $e_{m} \neq 0$ because 
$\theta_{m}\left(c_{m, 1}, \cdots, c_{m, k}\right)$ holds at some factor $A /(A \cap P)$ due to the fact that $\theta_{m}$ contains only one negation (just pick a factor in which $\psi_{m}\left(c_{m, 1}, \cdots, c_{m, k}\right)$ holds). Using the fact that there are no minimal idempotents in $A$ we can find disjoint idempotents $f_{m} \neq 0$ such that $\theta_{m}\left(c_{m, 1}, \cdots, c_{m, k}\right)$ holds identically on $f_{m}$. Then if

$$
c_{i}=\sum_{m=1}^{s} c_{m, i} f_{m}+\left(1-\bigcup f_{m}\right) c_{1, i}
$$

say, $A \vDash \varphi\left(c_{1}, \cdots, c_{k}\right)$, proving the claim.

Next we claim that it suffices to consider the case where $\varphi$ contains no negations at all. For consider $\theta=\bigwedge_{j=1}^{r} \varphi_{j} \wedge \psi$ such that $\prod A /(A \cap P) F$ $\exists x_{1} \cdots \exists x_{k} \theta$. If $\prod A /(A \cap P) \vDash \theta\left(b_{1}, \cdots, b_{k}\right)$ then $\theta\left(b_{1}, \cdots, b_{k}\right)$ holds in some factor $A /(A \cap P)$ because $\theta$ contains only one negation. Therefore since $A \rightarrow A /(A \cap P)$ is surjective there exist $c_{1}, \cdots, c_{k}$ in $A$ such that $\theta\left(c_{1}, \cdots, c_{k}\right)$ holds in the same factor. Therefore if $e$ is, by the Lemma, the idempotent in $A$ on which $\theta\left(c_{1}, \cdots, c_{k}\right)$ holds, $e \neq 0$; if we could find $d_{1}, \cdots, d_{k}$ in $A$ such that $A \vDash \bigwedge_{j=1}^{r} \varphi_{j}\left(d_{1}, \cdots, d_{k}\right)$, then we could set $x_{i}=e c_{i}+(1-e) d_{i}$ and conclude $A$ F $\theta\left(x_{1}, \cdots, x_{k}\right)$, finishing the proof.

So suppose that $\prod A /(A \cap P) \mid \exists \exists x_{1} \cdots \exists x_{k}\left(\bigwedge_{j=1}^{r} \varphi_{j}\right)$. Then $\bigwedge_{j=1}^{r} \varphi_{j}$ is solvable modulo any $(A \cap P)$. Now $\{A \cap P\}$, as $P$ varies over $\operatorname{Spec}(B)$, includes all maximal (=prime, by regularity) ideals in $A$. For given a prime ideal $Q$ in $A$, we claim that $Q B$ is a proper ideal in $B$. If not there exist $q_{1}, \cdots, q_{n}$ in $Q$ and $b_{1}, \cdots, b_{n}$ in $B$ such that $q_{1} b_{1}+\cdots+q_{n} b_{n}=1$; therefore, if $Q_{1}$ denotes the ideal in $A$ generated by $q_{1}, \cdots, q_{n}$, we have $Q_{1} B=B$. But $Q_{1}$ is principal (see [3]) with generator $q$, say. So $q B=B$, implying that $q$ is a unit and therefore $Q_{1}=A$, so $Q=A$, contradicting the properness of $Q$. Thus $Q B$ is a proper ideal in $B$ such that $Q B \cap A=Q$. If by Zorn's lemma we let $P$ be a proper ideal in $B$ which is maximal with respect to this property, then $P$ is a maximal ideal in $B$ by the maximality of $Q$ in $A$, and $A \cap P=Q$.

Thus $\bigwedge_{j=1}^{r} \varphi_{j}$ is solvable modulo all maximal ideals in $A$. We can assume $\varphi_{j}$ has the form $p_{j}=0$ for some polynomial $p_{j}$; then if for any $k$-tuple $\bar{b}=b_{1}, \cdots, b_{k}$ of elements of $A$ we let

$$
N_{(\bar{b})}=\left\{P \in \operatorname{Spec}(A) \mid p_{1}(\bar{b}), \cdots, p_{r}(\bar{b}) \in P\right\}
$$

$\left\{N_{(b)}\right\}$ covers $\operatorname{Spec}(A)$. Now

$$
N_{(b)}=\bigcap_{j=1}^{r}\left\{P \in \operatorname{Spec}(A) \mid p_{j}(\bar{b}) \in P\right\} ;
$$

if by regularity $c_{j}$ is an element of $A$ such that $p_{j}(\bar{b}) c_{j} p_{j}(\bar{b})=p_{j}(\bar{b})$, then 
$p_{j}(\bar{b}) c_{j}$ is an idempotent in $A$ and

$$
\begin{aligned}
\left\{P \in \operatorname{Spec}(A) \mid p_{j}(\bar{b}) \in P\right\} & =\left\{P \in \operatorname{Spec}(A) \mid p_{j}(\bar{b}) c_{j} \in P\right\} \\
& =\left\{P \in \operatorname{Spec}(A) \mid\left(1-p_{j}(\bar{b}) c_{j}\right) \notin P\right\},
\end{aligned}
$$

by the primeness of $P$ and the fact that $p_{j}(\bar{b})\left(1-p_{j}(\bar{b}) c_{j}\right)=0$. This last set is by definition an open set in the Zariski topology on $\operatorname{Spec}(A)$; hence each $N_{(b)}$ is open. By the compactness of the Zariski topology (see e.g. [3]), a finite number of $N_{(b)}$ 's cover $\operatorname{Spec}(A)$. It is now clear how to piece together $x_{1}, \cdots, x_{k}$ in $A$ such that $A \vDash \bigwedge_{j=1}^{r} \varphi_{j}\left(x_{1}, \cdots, x_{k}\right)$. This completes the proof.

For any set $S$ of primes let $\mathrm{Ch}(S)$ be the following set of axioms: $\{p$ is not invertible $\mid p \in S\} \cup\{p$ is invertible $\mid p \notin S\}$. For each infinite set of primes $S$ let $K_{S}^{\prime}=K^{\prime} \cup \mathrm{Ch}(S)$. For each finite set of primes $S$ let

$$
\begin{aligned}
& K_{S, 0}^{\prime}=K^{\prime} \cup \operatorname{Ch}(S) \cup\left\{\prod_{p \in S} p \neq 0\right\}, \\
& K_{S, 1}^{\prime}=K^{\prime} \cup \operatorname{Ch}(S) \cup\left\{\prod_{p \in S} p=0\right\} .
\end{aligned}
$$

THEOREM 2. The complete extensions of $K^{\prime}$ are precisely the $K_{S}^{\prime}$, $K_{S, 0}^{\prime}, K_{S, 1}^{\prime}$.

Proof. Define $K_{S}, K_{S, 0}, K_{S, 1}$ from $K$ in the same way that $K_{S}^{\prime}, K_{S, 0}^{\prime}$, $K_{S, 1}^{\prime}$ were defined from $K^{\prime}$. A trivial modification of the proof of Theorem 1 shows that $K_{S}, K_{S, 0}, K_{S, 1}$ have model companions $K_{S}^{\prime}, K_{S, 0}^{\prime}, K_{S, 1}^{\prime}$ respectively. To finish the proof it suffices to show that $K_{S}^{\prime}, K_{S, 0}^{\prime}, K_{S, 1}^{\prime}$ are complete because any complete extension of $K^{\prime}$ must extend one of these. To establish this completeness it suffices to show that all the $K_{S}$, $K_{S, 0}$, and $K_{S, 1}$ have the joint embedding property (see [6]).

First consider $K_{S, 0}$. By Lowenheim-Skolem considerations it suffices to consider two countable models $R_{1}$ and $R_{2}$ of $K_{S, 0}$. For each $p \in S \cup\{0\}$ let $F_{p}$ be the algebraically closed field of characteristic $p$ and transcendence degree $2^{\aleph_{0}}$. Let $R=\prod_{p \in S \cup\{0\}}\left(\prod_{i \in \omega} F_{p}\right)$. Then $R$ is a model of $K_{S, 0}$, and $R_{1}$ and $R_{2}$ can be embedded in $R$ since for $i=1,2$ at least one factor of characteristic $p$ occurs in the representation $R_{i} \rightarrow \prod_{P} R_{i} \mid P$, for each $p \in S \cup\{0\}$.

A similar argument without the factors of characteristic zero handles $K_{S, 1}$.

Finally consider $K_{S}, S$ infinite. Let $R$ be as above. Any countable model of $K_{S}$ is a subring of a countable product of $F_{p}$ 's, $p \in S$, or of $F_{p}$ 's, $p \in S \cup\{0\}$. The latter case may be handled as above. For the former case it suffices to show that $R_{1}=\prod_{p \in S}\left(\prod_{i \in \omega} F_{p}\right)$ can be embedded in $R$. 
Since $S$ is infinite $M=\{1,2,3, \cdots\} \subset R_{1}$ is a multiplicative set not containing zero. Let $P$ be a prime ideal in the complement of $M$. Then $R_{1} / P$ is an integral domain of characteristic zero, with quotient field a subfield of $F_{0}$. We have a homomorphism $\rho: R_{1} \rightarrow F_{0}$. Then the mapping which takes $\left\{x_{p, i}\right\}_{p \in S, i \in \omega} \in R_{1}$ to $\left\{\bar{x}_{p, i}\right\}_{p \in S \cup\{0\}, i \in \omega}$, where $\bar{x}_{0, i}=\rho(x)$ and $\bar{x}_{p, i}=$ $x_{p, i}$ for $p \in S$, is an embedding of $R_{1}$ into $R$.

REMARK. These results should be compared with the situation for fields, where we have as model companion the theory of algebraically closed fields, with complete extensions corresponding to the primes and zero.

THEOREM 3. $K^{\prime}$ is decidable.

Proof. If $\varphi$ is not a theorem of $K^{\prime}$ then $\neg \varphi$ is a theorem of some $K_{S}^{\prime}, K_{S, 0}^{\prime}$, or $K_{S, 1}^{\prime}$ and consequently a theorem of some finite extension of $K^{\prime}$ contained in some $K_{S}^{\prime}, K_{S, 0}^{\prime}$, or $K_{S, 1}^{\prime}$. Since $K^{\prime}$ is axiomatized, each of these finite extensions is axiomatized, and these finite extensions can be effectively listed, $K^{\prime}$ is decidable.

THEOREM 4. Let $K_{0}$ be the theory of commutative regular rings. Then $K^{\prime}$ is the model completion of $K_{0}$.

Proof. As in the proof of Theorem 1, since every regular ring is without nilpotents, $K_{0}$ and $K^{\prime}$ are mutually model consistent. Therefore $K^{\prime}$ is the model companion of $K_{0}$. Notice that $K^{\prime}$ extends $K_{0}$. Therefore to show that $K^{\prime}$ is the model completion of $K_{0}$ it suffices to show that $K_{0}$ has the amalgamation property (see [2]). This follows from a theorem of P. M. Cohn [1, Theorem 4.7].

REMARK. $K$ has no model completion, because the amalgamation property fails for $K$. For example let $S$ be the ring of all real valued continuous functions on $(0,1)$ which extend to continuous functions on $[0,1]$. Let $R_{1}$ be the ring of all real valued functions on $(0,1)$ and let $R_{2}$ be the ring of all real valued functions on $[0,1]$. Then $S \subset R_{1}$ and $S \subset R_{2}$. Consider the function $f(x)=x$ in $S . f$ is invertible in $R_{1}$ and is a zero divisor in $R_{2}$. Hence $R_{1}$ and $R_{2}$ cannot be amalgamated over $S$.

We also remark that the failure of the amalgamation property for $K$ implies that $K^{\prime}$ does not admit elimination of quantifiers. However if we augment the language by a function symbol $f$ and state the axiom of regularity in the form $\forall x(x f(x) x=x \wedge f(x) x f(x)=f(x))$, then $f(x)$ is uniquely determined by $x$ in any commutative regular ring, so $f(x)$ is definable in the unaugmented language, and therefore $K^{\prime}$ is model-complete in the new language (by the above), and hence the model completion of $K_{0}$ in the new language. But $K_{0}$ in the new language is a universal theory, 
so by a theorem of $\mathrm{A}$. Robinson [5], $K^{\prime}$ in the new language admits elimination of quantifiers.

\section{REFERENCES}

1. P. M. Cohn, On the free product of associative rings, Math. Z. 71 (1959), 380-398. MR 21 \#5648.

2. P. Eklof and G. Sabbagh, Model-completions and modules, Ann. Math. Logic 2 (1970/71), no. 3, 251-295. MR 43 \#3105.

3. J. Lambek, Lectures on rings and modules, Blaisdell, Waltham, Mass., 1966. MR 34 \#5857.

4. S. Lang, Algebra, Addison-Wesley, Reading, Mass., 1965. MR 33 \#5416.

5. A. Robinson, Introduction to the theory of models and metamathematics of algebra, North-Holland, Amsterdam, 1963. MR 27 \#3533.

6. - Infinite forcing in model theory, Proc. Second Scandinavian Logic Sympos., North-Holland, Amsterdam, 1971.

7. G. Sabbagh, Embedding problems for modules and rings with application to modelcompanions, J. Algebra 18 (1971), 390-403. MR 43 \#6259.

Department of Mathematics, Princeton University, Princeton, New Jersey 08540

Current address (L. Lipshitz): Department of Mathematics, Purdue University, Lafayette, Indiana 47907

Current address (D. Saracino): Department of Mathematics, Yale University, New Haven, Connecticut 06520 